\title{
Conference Paper \\ Hyaluronan Produced by Smooth Muscle Cells Plays a Critical Role in Neointima Formation
}

\author{
Davide Vigetti, Sara Deleonibus, Eugenia Karousou, Manuela Viola, \\ Giancarlo De Luca, and Alberto Passi \\ Dipartimento di Scienze Chirurgiche e Morfologiche, Università degli Studi dell'Insubria, Via J. H. Dunant 5, 21100 Varese, Italy
}

Correspondence should be addressed to Alberto Passi; alberto.passi@uninsubria.it

Received 6 November 2013; Accepted 9 March 2014; Published 12 May 2014

Academic Editors: V. La Carrubba, A. Lepedda, and J. C. Rodriguez-Cabello

This Conference Paper is based on a presentation given by Alberto Passi at "LIAC Meeting on Vascular Research 2013" held from 18 September 2013 to 21 September 2013 in Alghero, Italy.

Copyright (c) 2014 Davide Vigetti et al. This is an open access article distributed under the Creative Commons Attribution License, which permits unrestricted use, distribution, and reproduction in any medium, provided the original work is properly cited.

\begin{abstract}
Large body of evidence supports the idea that microenvironment plays a critical role in several pathologies including atherosclerosis and cancer. The amount of hyaluronan (HA) is involved in the microenvironment alterations and the concentration of this polymer reflects the progression of the diseases promoting neoangiogenesis, cell migration, and inflammation. The HA synthesis is regulated by several factors: UDP sugar precursors availability and the phosphorylation of synthetic enzyme HAS2 as well as specific drugs reducing the UDP precursors. The HAS2 phosphorylation is done by AMP kinase, a sensor of cell energy. When the cells have low energy, AMP kinase is activated and modifies covalently the regulatory enzymes, blocking all biosynthetic processes and activating the energy producing metabolism. It was recently reported that the hexosamine biosynthetic pathway (HBP) may increase the concentration of HA precursor UDP-N-acetylglucosamine (UDP-GlcNAc) leading to an increase of HA synthesis. We demonstrated that the increase of HA synthesis depends on the HAS2 post translational modification O-GlcNAcylation, which increases HA secretion modifying a residue different from the phosphorylation site of AMP kinase. In this report we highlighted the critical aspects of the post translational HAS2 regulation and its influence on HA synthesis.
\end{abstract}

\section{Introduction}

Cardiovascular diseases are among the major causes of premature death in modern society, and its impact is increasing due to rising rates of obesity and diabetes [1].

Chronic inflammation of the blood vessel wall in conjunction with dysfunctional lipoprotein homeostasis is the underlying cause of atherosclerosis. Limited success of pharmacological and invasive-surgical (i.e., angioplasty and bypass grafting) treatments may be a result of the incomplete understanding of the biological mechanisms which control and contribute to the development of atherosclerosis. In this context the extracellular matrix (ECM) components may play a critical role.

\section{Extracellular Matrix}

ECM not only gives the mechanical support to the cells, but also can interact with soluble factors (such as nutrients and signaling compounds) modulating the amount of molecules that reach the cells. Under this point of view, ECM remodeling due to physiological and pathological processes is critical to control cell response and the study of the ECM composition and that of enzymes involved in ECM remodeling (i.e., synthesis, degradation, and modification) can bring to the identification of new pivotal players controlling a particular event.

Among the different ECM components, glycosaminoglycans (GAGs) or mucopolysaccharides represent probably the most variable compounds. GAGs are composed of repeating disaccharide subunits that are assembled into linear polysaccharide chains. These polysaccharides are often covalently attached to proteoglycan proteins at the cell surface or in the ECM. There are several major classes of glycosaminoglycans, including heparan sulfate (HS) and heparin, chondroitin sulfate (CS), dermatan sulfate, and keratan sulfate, which differ in their core disaccharide subunit. HS and heparin contain 
d-N-acetyl-glucosamine (GlcNAc) and either d-glucuronic acid (GlcUA) or l-iduronic acid (IdoUA) subunits joined by $\alpha(1,4)$ and $\beta(1,4)$ linkages. CS has $N$-acetylgalactosamine (GalNAc) and GlcUA subunits and alternating $\beta(1,3)$ and $\beta(1,4)$ linkages.

The main function of GAGs is the maintenance of the support of the collagen and elastin and turgidity (bounce) in the cellular spaces, keeping those protein fibers in balance and proportion. It also promotes the ability of the collagen and elastin fibers to retain moisture and therefore remain soluble. In addition to these functions, which are merely linked to their chemical and physical properties, GAGs have specific effects on cells. Variations in GAGs relative amount, size, and modification have been described in several pathophysiological processes such as neuronal development, tumor growth and metastasis, viral invasion, spinal cord injury, and cardiovascular diseases.

GAGs, with the exception of hyaluronan, are assembled on core proteins by enzymes in the Golgi, using nucleotide sugars imported from the cytoplasm. During their assembly, they undergo a series of processing reactions involving $\mathrm{N}$-deacetylation, N-sulphation, epimerization of GlcUA to IdoUA, and O-sulphation that generate relatively short segments of modified sugars interspersed by variable tracts of unmodified sugars. More than 30 enzymes are required to properly synthesize GAGs and recent evidences suggest the presence of a complex multienzymatic structure dedicated to GAGs synthesis and processing into the Golgi named GAGosome. There is great structural heterogeneity in terms of chain length and size, the spacing of the modified tracts, and the extent of sulphation and epimerization within the modified segments. Processing also can occur by plasma-membranebound endosulfatases, which remove specific sulphate groups from the chains. Moreover, PGs and their GAGs on the cell surface can be shed by proteolytic cleavage of the core protein and by endoglycosidic cleavage of the GAGs chains by extracellular enzymes. In this way, bound ligands can be liberated and allowed to diffuse away from a cell.

Many factors determine the vascular disease progression but alterations in the extracellular matrix (ECM), which modifies the vascular cells microenvironment and induces abnormal cell behavior, are known to play a critical role in atherogenesis. Specifically, migration and growth of vascular smooth muscle cells (SMC) within the arterial intima coupled with deficient reendothelialization often lead to restenosis and/or thrombosis of treated arteries. In cardiovascular diseases there is an endothelial vascular damage that deeply compromises its functionality. Experimental and clinical data have shown a close link between endothelial dysfunction and inflammation. Proinflammatory cytokines are the primary responsible for the atheromatous plaque formation, the presence of which reduces vascular functionality. The endothelium is a functional barrier between the blood vessels and the blood stream, whose cells, by delimiting the vascular internal lumen, belong to complex system which modulates vascular tone, vascular smooth muscle cells, inflammation, and haemostasis, maintaining a proper blood sprinkling to tissues and regulating inflammatory mechanisms and coagulation [2]. The endothelium modulates vascular tone through several factors including nitric oxide (NO) and the development of vascular lesions is associated with a reduction of NO levels and increased amount of inflammatory markers [2].

\section{Hyaluronan Role}

Hyaluronan (HA) is quite different from other GAGs. In fact $\mathrm{HA}$ is a linear, unsulfated GAG that is composed of 2,000-25,000 disaccharides units of D-glucuronic acid and $\mathrm{N}$-acetylglucosamine linked together through alternating $\beta(1,4)$ and $\beta(1,3)$ glycosidic bonds. Differently from other sulfated GAGs that can show complex sulphation pattern, the information linked to HA is associated with the length of the polymer and, therefore, the amount and its molecular weight are important factors regulating the pathophysiological effects that this molecule displays on cells [2]. Moreover, HA is the only GAG to be not bounded to PG core protein and HA is synthesized by enzymes located in the cell plasma membrane that directly extruded it in the extracellular space [3]. The mechanisms controlling HA synthesis and molecular assembly are yet not completely known. HA catabolism in the ECM is attributed to various hyaluronidases and its half-life in mammalian tissues may vary from one day in the skin to up to 2-3 weeks in the cartilage [4].

ECMs in which HA is abundant are very hydrated creating an ideal environment in which cells can move, proliferate, and receive nutrients and other signaling molecules. Moreover, HA is an important space filling molecule as is evident in the vitreous humor, the dermis, and the synovial fluid of joints. HA content differs in tissues depending on the type and the metabolic situation, being greater in foetal and cicatricial tissues $[5,6]$ and in very early tissue remodelling $[7,8]$ suggesting its major role in tissue repair processes. In addition, it has been shown that intact HA molecule may act as a free radical scavenger [9].

Besides its chemical and mechanical properties, HA interacts with several receptors at the cellular level that specifically trigger various signal transduction responses [10]. The HA receptor CD44 is expressed on the surface of most cells, including immune system cells, and it mediates cell adhesion, proliferation, and migration [11]. Receptor for HA-mediated motility (RHAMM) mediates cellular motility [12]. Lyve-1 is the specific HA receptor of the lymphatic system although very recent evidences indicate a more complex function of this protein unrelated to HA [13]. HA receptor for endocytosis (HARE) mediates the endocytosis of HA $[14,15]$. Recently, Toll-like receptors 4 and 2 (TLR4/2) were shown to recognize HA fragments [16] and modulate the inflammation response in the lung [17]. Although it has a very simple structure, it is clear that HA can modulate many cellular responses, and the amount of this GAG has to be strictly regulated.

In mammals, HA synthesis depends on the activity of three different enzymes called HA synthases (HAS1, 2, and 3). In other animals this enzymatic pattern seems conserved; for instance in our laboratory we were able to isolate in Xenopus laevis the three hyaluronan synthases, cloning HAS3 for the first time [18]. Nevertheless, the regulation of HA synthases activity is still unclear; we reported that substrates (UDP 
sugars) availability plays an important role in inducing HAS transcription in human cells [19].

Hyaluronic acid (HA), a prevalent component of the extracellular and pericellular matrixes, plays a key role in several biological processes [20]. Although the HA function and extracellular organization were deeply examined in a wide number of microenvironments, its presence in the endothelium was not fully explained. The binding between HA and the cell surface is mediated by HA-binding proteins, such as CD44 and receptor for HA-mediated motility (RHAMM), of which CD44 is considered as a major HA cell surface receptor [21]. Notably, generation of an active, HA-binding form of this receptor is responsible for most of CD44's biological functions, such as myelo- and leukopoiesis and leukocyte extravasation and activation [10]. There is an emerging evidence that HA associated with vessel wall contributes to targeting lymphocytes to inflammatory sites [22]. In fact, HA on endothelial cells can initiate contact and mediate rolling of circulating cells bearing active form of CD44 [22]. The involvement of the CD44-HA complex in promoting chronic inflammatory conditions is also supported by findings showing that administration of anti-CD44 antibodies, which specifically blocks the CD44-HA interactions, results in a reduction of inflammatory symptoms such as $\mathrm{T}$ cell infiltrates and tissue oedema in a number of experimental models of inflammation [12-14]. Indeed, CD44-mediated HA binding has been implicated in the adhesion of a leukemic cell line to vascular endothelial cells [15]. As the HA-mediated interactions between endothelium and leukocytes or disseminating tumor cells play an important role in extravasation of circulating cells, there is a great need to determine the potential of endothelial cells to express HA as well as the need for identifying mechanisms underlying HA-mediated migration across vascular endothelium.

It has been reported that some inflammatory cytokines, such as tumor necrosis factor-alpha, (TNF-alpha), interleukin 1-beta (IL-1beta), and interferon-gamma (IFN-gamma), can differently induce and modulate HASes expression in human skin fibroblasts $[16,17]$. Analogue results were obtained using endothelial cell cultures treated with TNFalpha, IL-1beta, IL-15, and lipopolysaccharide (LPS) $[4,8,9]$. In addition, this modulation seems to be closely restricted to endothelial cells derived from microvasculature and not from big vessels, coherently with a fundamental role played in the vasculature where the most leukocyte traffic occurs. The increased HA levels induced by cytokines may also facilitate adhesive interactions between leukocytes and endothelial cells.

The HA also appears to have a direct role in the modulation of the inflammatory mechanism. It has been shown that HA, in relation to its molecular weight, can play a dual action. Low molecular weight HA seems to promote inflammation, whereas high molecular weight HA seems to inhibit inflammation. The inflammatory action occurs through activation, by small fragments of HA, of the CD44 and TLR- 4 receptors $[8,9]$. For TLR-4, the anti-inflammatory action is due to long chains of polymerized HA directly blocking the TLR- 4 receptor, therefore, preventing the fact that fragments of HA may bind TLR-4 [8]. For CD44 the high molecular weight HA does not seem to target directly the receptor, rather a product of its activation, the protein kinase $\mathrm{C}(\mathrm{PKC})[8]$. It appears that the fragments resulting from HA degradation/depolymerization produced by the chondroitinase, hyaluronidase, and ROS, in the initial phase of inflammation, may increase and amplify inflammation through the direct stimulation of the receptors CD44 and TLR-4 [8]. This stimulation is able to induce protein mediator activation, such as some isoforms of PKC (particularly PKCdelta and PKCepsilon) following CD44 stimulation, and the myeloid differentiation factor 88 (MyD88) and the TNF associated factor (TRAF-6) following TLR-4 stimulation, all together converge in the activation of NF- $\kappa \mathrm{B}[18,23]$. The activation of NF- $\kappa$ B in turn produces the transcription of a series of inflammatory mediators such as proinflammatory cytokines and metalloproteases (MMPs) that amplify inflammation and apoptotic precursors that lead to cell death [24, 25]. Several reports have shown that the proinflammatory cytokines and MMPs, produced by activated cells, play a key role in cardiovascular disease.

The secreted product of tumour necrosis factor-stimulated gene-6 (TSG-6) is an HA-binding protein, the expression of which is very tightly regulated [26]. There is little or no constitutive expression of TSG-6 in adult tissues, but the protein is synthesized by fibroblasts, chondrocytes, monocytes, and vascular endothelial and smooth muscle cells in response to stimulation with proinflammatory mediators or certain growth factors. It has been suggested that TSG6 might modulate cellular interactions with $\mathrm{HA}$ at sites of inflammation [27]. The HA binding domains of TSG-6 and CD44 both contain a "link module," a structural unit of about 100 amino acids that is present in many other members of the hyaladherin family including the proteoglycan aggrecan [28] and cartilage link protein $[26,29,30]$. Although TSG- 6 binds HA with high affinity [31], the binding between CD44 and HA is much weaker [32] and seems to depend on multivalent interactions [33]. However, recent studies have shown the involvement, in this mechanism, of a protein factor called inter-alpha-inhibitor (IaI). IaI comprises a light chain which may exist in a free form called bikunin, which is a serine protease inhibitor, and at least six closely related heavy chains. Among them the heavy chains called $\mathrm{HCl}$ and $\mathrm{HC} 2$ are the most studied [33]. In vitro studies have shown that these chains, $\mathrm{H1}$ and $\mathrm{H} 2$, form covalent binding with TSG-6 or HA. The TSG-6-heavy chains (TSG-6-HC1 and TSG-6-HC2) act as intermediates during $\mathrm{HCl}$ and $\mathrm{HC} 2$ transfer from IaI to $\mathrm{HA}$ in order to form HC-HA complex. TSG-6, which is released upon the transfer of HCs from TSG-6 onto HA, was shown to combine with IaI to generate new TSG-6-HC complexes and thus acts as a true catalyst for the formation of HC-HA [32].

\section{Conclusion}

In relation to vascular pathologies, SMC and endothelial cells have a pivotal role in neointima formation and HA plays a critical role in this process. In fact, the intima thickening is determined by SMC proliferation and migration from the tunica media, which are strongly dependent on HA content. One of the earliest events is SMC dedifferentiation from 
contractile to synthetic phenotype. In this latter state SMC start to synthesize a large amount of ECM components such as GAGs and HA, particularly after oxidized LDL exposure [34]. Moreover, EC can modulate immune cells infiltration by regulating the rolling and the extravasation of monocytes and leucocytes. Although this event is mainly controlled by adhesion molecules like integrins and selectins, HA is to be considered a new player in inflammation as it was discovered that immune cells can interact with HA synthesized by EC through CD44. In fact the inflamed tissue develops a dramatic increase of HA and the HA overproduction occurs at the very early phase of the process, as the first step of the pathological overproduction of ECM components. Therefore, ECM remodeling represents a crucial event in the onset of vascular diseases and to know how the synthesis of such GAGs is regulated could bring to the identification of new molecular target for vasoprotective drugs.

\section{Conflict of Interests}

The authors declare that there is no conflict of interests regarding the publication of this paper.

\section{References}

[1] P. N. Hopkins, "Molecular biology of atherosclerosis," Physiological Reviews, vol. 93, no. 3, pp. 1317-1542, 2013.

[2] C. Zhang, "The role of inflammatory cytokines in endothelial dysfunction," Basic Research in Cardiology, vol. 103, no. 5, pp. 398-406, 2008.

[3] Y. Yamada, N. Itano, K.-I. Hata, M. Ueda, and K. Kimata, "Differential regulation by IL-1beta; and EGF of expression of three different hyaluronan synthases in oral mucosal epithelial cells and fibroblasts and dermal fibroblasts: quantitative analysis using real-time RT-PCR," Journal of Investigative Dermatology, vol. 122, no. 3, pp. 631-639, 2004.

[4] G. M. Campo, A. Avenoso, S. Campo, D. Angela, A. M. Ferlazzo, and A. Calatroni, "TNF- $\alpha$, IFN- $\gamma$, and IL- $1 \beta$ modulate hyaluronan synthase expression in human skin fibroblasts: Synergistic effect by concomital treatment with FeSO 4 plus ascorbate," Molecular and Cellular Biochemistry, vol. 292, no. 12, pp. 169-178, 2006.

[5] M. Mohamadzadeh, H. DeGrendele, H. Arizpe, P. Estess, and M. Siegelman, "Proinflammatory stimuli regulate endothelial hyaluronan expression and CD44/HA-dependent primary adhesion," Journal of Clinical Investigation, vol. 101, no. 1, pp. 97108, 1998.

[6] P. Estess, A. Nandi, M. Mohamadzadeh, and M. H. Siegelman, "Interleukin 15 induces endothelial hyaluronan expression in vitro and promotes activated $\mathrm{T}$ cell extravasation through a CD44-dependent pathway in vivo," Journal of Experimental Medicine, vol. 190, no. 1, pp. 9-19, 1999.

[7] A. Nandi, P. Estess, and M. H. Siegelman, "Hyaluronan anchoring and regulation on the surface of vascular endothelial cells is mediated through the functionally active form of CD44," Journal of Biological Chemistry, vol. 275, no. 20, pp. 1493914948, 2000.

[8] G. M. Campo, A. Avenoso, S. Campo, A. D’Ascola, P. Traina, and A. Calatroni, "Differential effect of molecular size HA in mouse chondrocytes stimulated with PMA," Biochimica et Biophysica Acta, vol. 1790, no. 10, pp. 1353-1367, 2009.
[9] G. M. Campo, A. Avenoso, S. Campo, A. D’Ascola, G. Nastasi, and A. Calatroni, "Molecular size hyaluronan differently modulates toll-like receptor-4 in LPS-induced inflammation in mouse chondrocytes," Biochimie, vol. 92, no. 2, pp. 204-215, 2010.

[10] J. Cichy and E. Puré, "The liberation of CD44," Journal of Cell Biology, vol. 161, no. 5, pp. 839-843, 2003.

[11] M. H. Siegelman, D. Stanescu, and P. Estess, "The CD44initiated pathway of T-cell extravasation uses VLA-4 but not LFA-1 for firm adhesion," Journal of Clinical Investigation, vol. 105 , no. 5, pp. 683-691, 2000.

[12] K. Mikecz, F. R. Brennan, J. H. Kim, and T. T. Glant, "Anti-CD44 treatment abrogates tissue oedema and leukocyte infiltration in murine arthritis," Nature Medicine, vol. 1, no. 6, pp. 558-563, 1995.

[13] S. Brocke, C. Piercy, L. Steinman, I. L. Weissman, and T. Veromaa, "Antibodies to CD44 and integrin $\alpha 4$, but not L-selectin, prevent central nervous system inflammation and experimental encephalomyelitis by blocking secondary leukocyte recruitment," Proceedings of the National Academy of Sciences of the United States of America, vol. 96, no. 12, pp. 6896-6901, 1999.

[14] L. Weiss, S. Slavin, S. Reich et al., "Induction of resistance to diabetes in non-obese diabetic mice by targeting CD44 with a specific monoclonal antibody," Proceedings of the National Academy of Sciences of the United States of America, vol. 97, no. 1, pp. 285-290, 2000.

[15] A. Maiti, G. Maki, and P. Johnson, "TNF- $\alpha$ induction of CD44mediated leukocyte adhesion by sulfation," Science, vol. 282, no. 5390, pp. 941-943, 1998.

[16] T. Oguchi and N. Ishiguro, "Differential stimulation of three forms of hyaluronan synthase by TGF- $\beta$, IL- $1 \beta$, and TNF- $\alpha$," Connective Tissue Research, vol. 45, no. 4-5, pp. 197-205, 2004.

[17] Y. Yamada, N. Itano, K.-I. Hata, M. Ueda, and K. Kimata, "Differential regulation by IL-1beta; and EGF of expression of three different hyaluronan synthases in oral mucosal epithelial cells and fibroblasts and dermal fibroblasts: quantitative analysis using real-time RT-PCR," Journal of Investigative Dermatology, vol. 122, no. 3, pp. 631-639, 2004.

[18] P. Heldin, E. Karousou, B. Bernert, H. Porsch, K. Nishitsuka, and S. Skandalis, "Importance of hyaluronan-CD44 interactions in inflammation and tumorigenesis," Connective Tissue Research, vol. 49, no. 3-4, pp. 215-218, 2008.

[19] D. Vigetti, M. Ori, M. Viola et al., "Molecular cloning and characterization of UDP-glucose dehydrogenase from the amphibian Xenopus laevis and its involvement in hyaluronan synthesis," Journal of Biological Chemistry, vol. 281, no. 12, pp. 8254-8263, 2006.

[20] J. Y. Lee and A. P. Spicer, "Hyaluronan: a multifunctional, megaDalton, stealth molecule," Current Opinion in Cell Biology, vol. 12, no. 5, pp. 581-586, 2000.

[21] H. Ponta, L. Sherman, and P. A. Herrlich, "CD44: from adhesion molecules to signalling regulators," Nature Reviews Molecular Cell Biology, vol. 4, no. 1, pp. 33-45, 2003.

[22] M. H. Siegelman, H. C. DeGrendele, and P. Estess, "Activation and interaction of CD44 and hyaluronan in immunological systems," Journal of Leukocyte Biology, vol. 66, no. 2, pp. 315321, 1999.

[23] K. J. Loniewski, S. Patial, and N. Parameswaran, "Sensitivity of TLR4- and -7-induced NFאB1 p105-TPL2-ERK pathway to TNF-receptor-associated-factor- 6 revealed by RNAi in mouse macrophages," Molecular Immunology, vol. 44, no. 15, pp. 37153723, 2007. 
[24] G. Murphy and H. Nagase, "Progress in matrix metalloproteinase research," Molecular Aspects of Medicine, vol. 29, no. 5, pp. 290-308, 2009.

[25] R. K. Winn and J. M. Harlan, "The role of endothelial cell apoptosis in inflammatory and immune diseases," Journal of Thrombosis and Haemostasis, vol. 3, no. 8, pp. 1815-1824, 2005.

[26] C. M. Milner and A. J. Day, "TSG-6: A multifunctional protein associated with inflammation," Journal of Cell Science, vol. 116, no. 10, pp. 1863-1873, 2003.

[27] J. D. Kahmann, R. O’Brien, J. M. Werner et al., "Localization and characterization of the hyaluronan-binding site on the Link module from human TSG-6," Structure, vol. 8, no. 7, pp. 763774, 2000.

[28] M. J. Merrilees, B. W. Beaumont, K. R. Braun et al., "Neointima formed by arterial smooth muscle cells expressing versican variant V3 is resistant to lipid and macrophage accumulation," Arteriosclerosis, Thrombosis, and Vascular Biology, vol. 31, no. 6, pp. 1309-1316, 2011.

[29] C. D. Blundell, D. J. Mahoney, A. Almond et al., "The link module from ovulation- and inflammation-associated protein TSG-6 changes conformation on hyaluronan binding," The Journal of biological chemistry, vol. 278, no. 49, pp. 49261-49270, 2003.

[30] P. Teriete, S. Banerji, M. Noble et al., "Structure of the regulatory hyaluronan binding domain in the inflammatory leukocyte homing receptor CD44," Molecular Cell, vol. 13, no. 4, pp. 483496, 2004.

[31] J. Lesley, N. M. English, I. Gál, K. Mikecz, A. J. Day, and R. Hyman, "Hyaluronan binding properties of a CD44 chimera containing the link module of TSG-6," Journal of Biological Chemistry, vol. 277, no. 29, pp. 26600-26608, 2002.

[32] L. Zhuo and K. Kimata, "Structure and function of inter- $\alpha$ trypsin inhibitor heavy chains," Connective Tissue Research, vol. 49, no. 5, pp. 311-320, 2008.

[33] M. S. Rugg, A. C. Willis, D. Mukhopadhyay et al., "Characterization of complexes formed between TSG- 6 and inter- $\alpha$-inhibitor that act as intermediates in the covalent transfer of heavy chains onto hyaluronan," Journal of Biological Chemistry, vol. 280, no. 27, pp. 25674-25686, 2005.

[34] M. Viola, B. Bartolini, D. Vigetti et al., "Oxidized low density lipoprotein (LDL) affects hyaluronan synthesis in human aortic smooth muscle cells," The Journal of Biological Chemistry, vol. 288, pp. 29595-29603, 2013. 


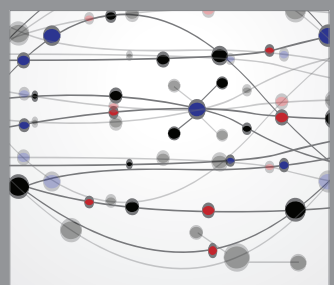

The Scientific World Journal
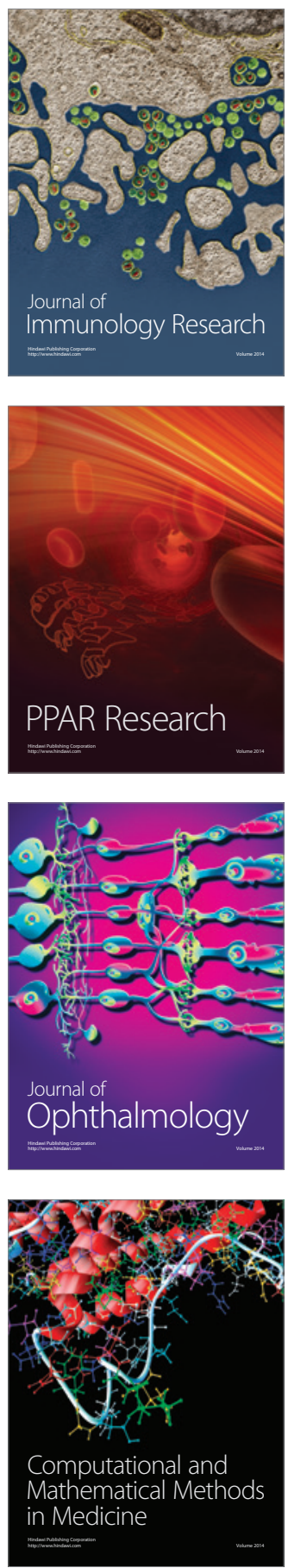

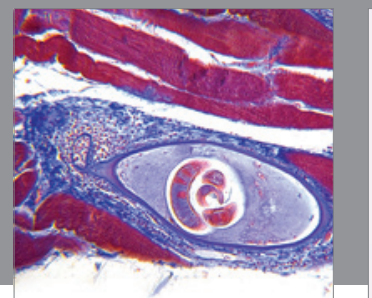

Gastroenterology

Research and Practice
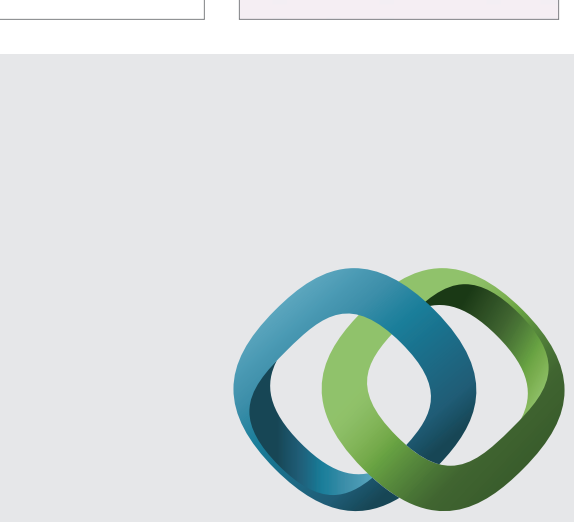

\section{Hindawi}

Submit your manuscripts at

http://www.hindawi.com
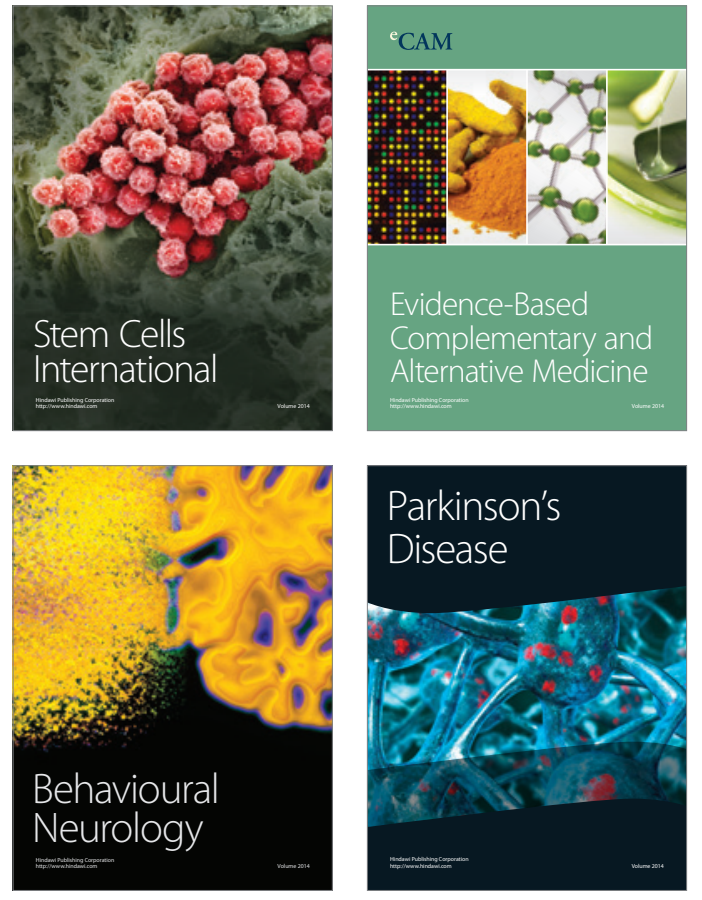
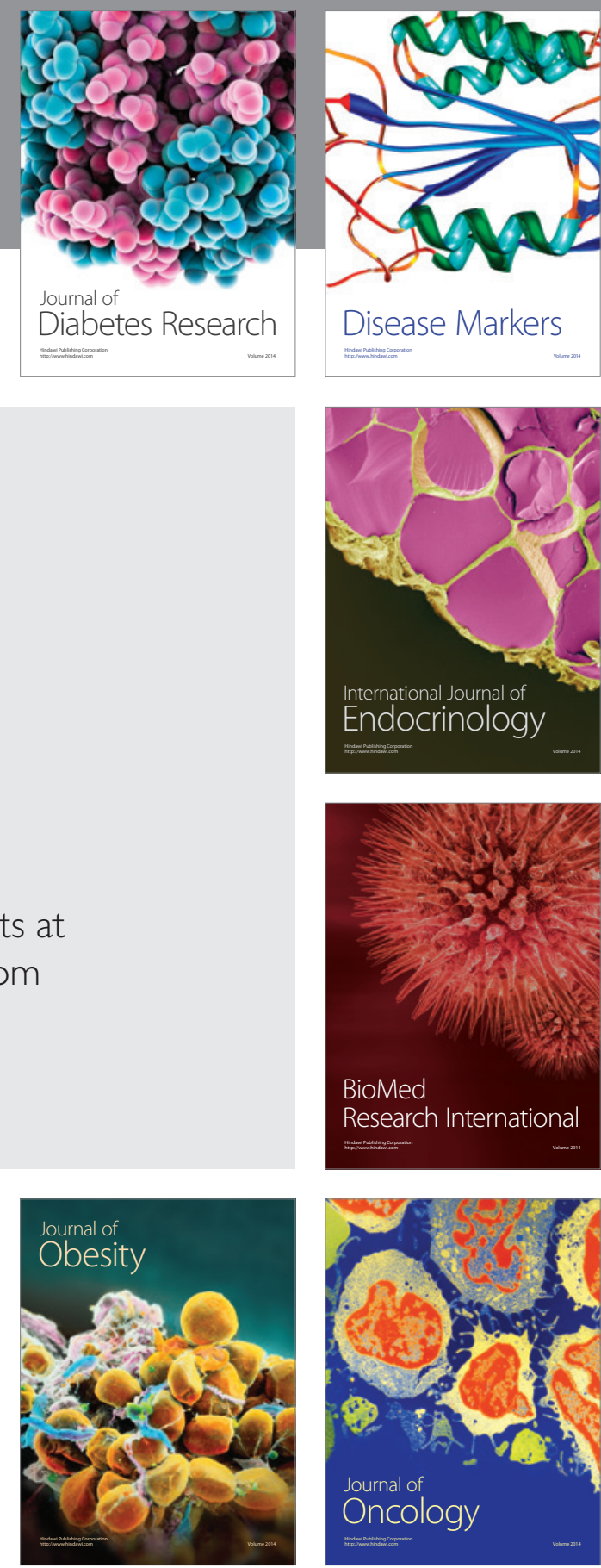

Disease Markers
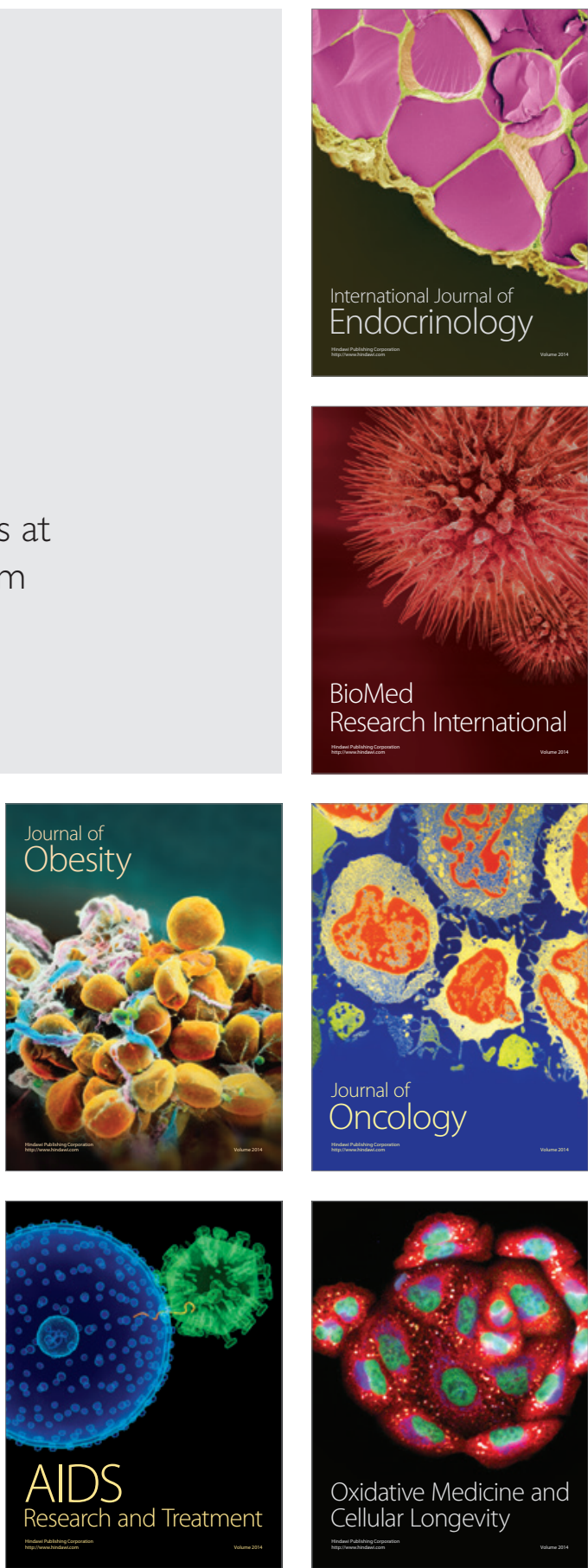